\title{
SMLTM simulations of the diurnal tide: comparison with UARS observations
}

\author{
R. A. Akmaev ${ }^{1}$, V. A. Yudin $^{2}$, D. A. Ortland ${ }^{3}$ \\ ${ }^{1}$ Department of Aerospace Engineering Sciences, Campus Box 429, University of Colorado, Boulder, CO 80309-0429, USA \\ ${ }^{2}$ Institute for Terrestrial and Planetary Atmospheres, State University of New York at Stony Brook, Stony Brook, NY 11794-5000, USA \\ ${ }^{3}$ Space Physics Research Laboratory, University of Michigan, 2455 Hayward Street, Ann Arbor, MI 48109-2143, USA
}

Received: 25 September 1996 / Revised: 6 January 1997 / Accepted: 9 January 1997

\begin{abstract}
Wind and temperature observations in the mesosphere and lower thermosphere (MLT) from the Upper Atmosphere Research Satellite (UARS) reveal strong seasonal variations of tides, a dominant component of the MLT dynamics. Simulations with the Spectral mesosphere/lower thermosphere model (SMLTM) for equinox and solstice conditions are presented and compared with the observations. The diurnal tide is generated by forcing specified at the model lower boundary and by in situ absorption of solar radiation. The model incorporates realistic parameterizations of physical processes including various dissipation processes important for propagation of tidal waves in the MLT. A discrete multi-component gravity-wave parameterization has been modified to account for seasonal variations of the background temperature. Eddy diffusion is calculated depending on the gravitywave energy deposition rate and stability of the background flow. It is shown that seasonal variations of the diurnal-tide amplitudes are consistent with observed variations of gravity-wave sources in the lower atmosphere.
\end{abstract}

\section{Introduction}

Atmospheric solar tides are global-scale waves permanently forced by thermal excitation due to absorption of solar radiation at various levels in the atmosphere. Tidal waves generated in the troposphere and stratosphere encounter little dissipation propagating upward into the mesosphere and lower thermosphere (MLT) region. Large horizontal phase speeds minimize the effects of background (zonal mean) winds on tidal propagation. Due to exponential decrease in atmospheric density their

Correspondence to: R. A. Akmaev amplitudes grow substantially and tides become a dominant feature of the MLT dynamics (e.g., Forbes, 1995). In the MLT region various dissipation processes can substantially affect the propagation of tidal waves (e.g., Forbes, 1982; Hagan et al., 1995). Momentum and energy deposition and eddy mixing generated by dissipating and breaking gravity waves, molecular viscosity and thermal conductivity are most important dissipation mechanisms. Recent global observations of MLT winds by the High-resolution Doppler imager (HRDI) and Wind-imaging interferometer (WINDII) from the Upper Atmosphere Research Satellite (UARS) (e.g., Hays et al., 1994; McLandress et al., 1994) confirm the dominant role of tides. The observations also reveal strong seasonal variations of tidal amplitudes. In particular, the strong diurnal tide at subtropical latitudes undergoes a pronounced semi-annual cycle with maximum amplitudes at equinox and minima of about a factor of 2 smaller at solstice (Hays et al., 1994; Burrage et al., 1995; McLandress et al., 1996). The observed seasonal variations of tidal amplitudes cannot be fully explained by seasonal variations of tidal forcing in the lower atmosphere (Groves, 1982a, b), of the background zonal mean circulation, or of the molecular diffusivity and viscosity (e.g., Burrage et al., 1995; Hagan et al., 1995; Hagan, 1996). This suggests that seasonal variations of dissipation induced by gravity waves may play an important role (e.g., Burrage et al., 1995; Khattatov et al., 1997).

Small-scale gravity waves generated by various dynamical processes in the lower atmosphere penetrate into the MLT where they deposit their momentum and energy and produce turbulence due to dissipation and breaking (e.g., Lindzen, 1981; Holton, 1982; Matsuno, 1982; Hines, 1997). The wave drag and mixing control the large-scale dynamics and climatology in the middle and upper atmosphere to a great extent (e.g., Akmaev et al., 1992; Akmaev, 1994). There are numerous indications that at low and middle latitudes the smallscale gravity-wave activity (e.g., Nakamura et al., 1996) and related eddy mixing (e.g., Fukao et al., 1994) exhibit 
a strong seasonal cycle in the MLT region with solstitial maxima and equinoctial minima. Gravity waves propagating upward from sources in the lower atmosphere are much more sensitive than tides to the background wind and temperature distributions. Eckermann (1995) considered annual variations of gravity waves due to only seasonal variations of temperature stratification in the stratosphere. He was able to reproduce some observed features of the annual cycle of gravity wave activity at $60 \mathrm{~km}$ in a windless atmosphere neglecting possible variations of wave sources. Obviously, variations in source strengths and large-scale background winds should also contribute to the observed seasonal cycle of gravity waves. For example, the observations analyzed by Allen and Vincent (1995) show considerable seasonal variations in gravity-wave energy density in the lower atmosphere that are generally consistent with the maxima observed in the MLT at solstice.

Akmaev et al. (1996) presented a new spectral mesosphere/lower thermosphere model (SMLTM). The model is an extension into the thermosphere of the three-dimensional middle-atmospheric model developed by Akmaev et al. (1992). The original gravity-wave parameterization with a uniform and isotropic forcing at the lower boundary (Akmaev et al., 1992; Akmaev, 1994) was optimized to better reproduce the empirical climatology of the middle atmosphere (Fleming et al., 1990). Akmaev et al. (1996) used the eddy-diffusion coefficient independently specified from the global thermal balance according to the procedure described by Akmaev (1994). The model produced simulations of the diurnal tide at equinox in close agreement with the UARS climatology (McLandress et al., 1996). In this study, the work by Akmaev et al. (1996) is extended to reproduce the observed variations of the diurnal-tide amplitudes from equinox to solstice. The original gravity-wave parameterization by Akmaev et al. (1992) and Akmaev (1994) is modified to account for background-temperature variations. Unlike in the previous work, here the coefficient of eddy diffusion is calculated self-consistently depending on the gravity-wave energy deposition rate and background stability. The gravitywave source strength is allowed to vary with latitude and season to mimic the gross features of the climatology by Allen and Vincent (1995). It is shown that with these modifications the model is capable of reproducing the seasonal variations of the diurnal-tide amplitude in reasonable agreement with the UARS observations.

The model is briefly introduced in Sect. 2 followed by a description of modifications of gravity-wave and eddymixing calculations in Sect. 3. Section 4 presents simulations of the diurnal tide for equinox and solstice conditions and comparisons with UARS observations.

\section{Model}

For more than two decades three-dimensional nonlinear spectral general circulation models (GCMs) (e.g., Bourke et al., 1977) have been successfully employed in numerical weather prediction and climate studies in the lower atmosphere. The spectral method, wherein model scalar variables are naturally represented in terms of truncated series in spherical harmonics, appears to be even more appropriate for study of global-scale wave phenomena. This is why computationally efficient models based on the spectral platform gain popularity in the upper atmospheric research as well. Wu et al. (1989) and Miyahara et al. (1993) extended a tropospheric spectral GCM into the lower thermosphere to study nonlinear interactions of tides and gravity waves with the mean flow. Chan et al. (1994) presented a middle- and upper-atmospheric model based on a spectral formulation that differs in some details from the traditional one (Bourke et al., 1977) but still takes advantage of the readily implementable semi-implicit time-integration scheme. Using this model in a twodimensional configuration Mengel et al. (1995) studied equatorial oscillations in the middle atmosphere generated by gravity waves.

Akmaev et al. (1992) developed a spectral middleatmospheric $(\sim 15-120 \mathrm{~km})$ model that has since been used both in two- and three-dimensional configurations for simulations and diagnostics of the zonal mean climatology (Akmaev, 1994; Akmaev, 1997). Recently the model was extended into the thermosphere to prevent spurious reflections of strong tidal waves from the upper boundary (Akmaev et al., 1996). The present version, the SMLTM, has the same horizontal resolution as the middle-atmospheric version approximately corresponding to $9^{\circ} \times 9^{\circ}$ in latitude-longitude. The model vertical domain is divided into 38 layers of $1 / 2$ pressure scale height each and extends from the lower boundary at $100 \mathrm{mb}$ to the upper boundary at about $220 \mathrm{~km}$. Tropospheric tidal forcing is introduced via the lower-boundary condition. Diurnal and semidiurnal variations of horizontal wind components, temperature, and geopotential at $100 \mathrm{mb}$ (nominal altitude $16.3 \mathrm{~km}$ ) are specified as functions of latitude and local time according to simulations with the Global-scale wave model (GSWM) (Hagan et al., 1995). No Hough-mode decomposition is applied. Currently, the GSWM simulations are available for January, April, July, and October, representing the four seasons. In this study, we present simulations for the northern-hemisphere spring and winter conditions.

A variety of physical processes important in the middle and upper atmosphere are represented in the model. The parameterization by Strobel (1978) with modifications by Apruzese et al. (1982) and the parameterization by Shine and Rickaby (1989) are used for calculations of ultraviolet solar heating due to absorption by $\mathrm{O}_{2}$ and $\mathrm{O}_{3}$, respectively. The absorption of solar extreme ultraviolet (EUV) radiation is calculated using absorption cross sections for $\mathrm{O}, \mathrm{O}_{2}$, and $\mathrm{N}_{2}$ by Torr et al. (1979) and a revised version of the solar EUV flux model by Tobiska (1991). The infrared radiation transfer in the 9.6- $\mu \mathrm{m} \mathrm{O}_{3}$ and $15-\mu \mathrm{m} \mathrm{CO}_{2}$ bands is treated according to Fomichev and Shved (1985) and Fomichev et al. (1993), respectively. The coefficients of molecular viscosity and thermal conductivity are calculated according to Banks and Kockarts (1973, §14.3). Daily 
mean ion drag coefficients (e.g., Forbes, 1982) are calculated using the IRI-90 empirical ionospheric model (Bilitza, 1990). The discrete-spectrum gravity-wave parameterization based on the formulation by Gavrilov (1990) is described by Akmaev et al. (1992) and Akmaev (1994). The next section presents some modifications introduced into the gravity-wave scheme in this study and the new procedure for eddy-mixing calculations.

Further details of the model implementation can be found in the papers by Akmaev et al. (1992) and Akmaev (1994).

\section{Gravity waves and eddy mixing}

\subsection{Gravity-wave drag and heating}

The discrete-spectrum gravity-wave parameterization based on the formulation by Gavrilov (1990) is described in detail by Akmaev et al. (1992). Here we only reproduce the equations that will be needed later and mention some modifications introduced in this study. The horizontal momentum deposition rate or acceleration of the background flow by a single monochromatic wave $i$ is calculated as

$a_{i}=-\frac{1}{\rho} \frac{\partial \rho F_{i}}{\partial z}$

where $\rho$ is atmospheric density, $F_{i}=\delta_{i} f_{i}$ effective vertical flux of horizontal momentum, $\delta_{i}$ "efficiency factor" (e.g., Holton, 1983), and $f_{i}$ vertical flux of horizontal momentum. Away from the breaking level, $f_{i}$ is determined from the equation

$\frac{1}{\rho} \frac{\partial \rho f_{i}}{\partial z}=-\alpha_{i} f_{i}$,

where $\alpha_{i}$ is the absorption coefficient depending on coefficients of eddy and molecular viscosity and thermal conductivity and radiative damping (Gavrilov, 1990; Akmaev et al., 1992). Equation 2 is a generalized equivalent of the WKB "transmission function" formulation by Plumb and McEwan (1978) (see also, Matsuno, 1982).

There is no special treatment above or below the breaking level. For each wave Eq. 2 is numerically solved at consecutive model levels starting from the lower boundary. At every model level the effective vertical flux $F_{i}$ is compared with its "breaking" value

$F_{i b}=\delta_{i} k_{i}\left(c_{i}-u_{i}\right)^{3} / 2 N$,

where $k_{i}$ is the horizontal wavenumber, $c_{i}$ horizontal phase speed, $u_{i}$ projection of the background wind onto the wave propagation direction, and $N$ buoyancy frequency. From the two values of $F_{i}$, the one with the minimal absolute value is selected. Thus a wave is assumed breaking when it reaches breaking conditions, i.e., when $F_{i}$ is calculated according to Eq. 3. In principle, it is possible for the wave to return to "normal" dissipative propagation (Eq. 2) if dissipation becomes strong enough above the region of breaking. This assumption appears to be confirmed by recent numerical simulations of gravity-wave propagation and breaking by Prusa et al. (1996), in which laminar wave propagation above the breaking regime can be clearly observed. If $F_{i}$ changes sign between two adjacent model levels or turns to 0 at some level, it is assumed that a critical level has been reached and $F_{i}$ is set to 0 at the current and all overlying levels. Waves that do not encounter critical levels or dissipate in the mesosphere eventually entirely dissipate in the thermosphere due to strong molecular viscosity and thermal conductivity. The wave $\operatorname{drag} a_{i}$ is calculated from the vertical profile of $F_{i}$ according to Eq. 1 .

Akmaev (1994) derived the following expression for the part of total wave energy loss (Gavrilov, 1990) available for dissipation:

$\epsilon_{i}=\left(c_{i}-u_{i}\right) a_{i}$

An equivalent expression for a single monochromatic wave is used by Hines (1997). Note that the drag $a_{i}$ is always in the direction of the intrinsic phase speed, $\left(c_{i}-u_{i}\right)$, and therefore $\epsilon_{i} \geq 0$.

The total wave drag and dissipation (heating) are calculated as a vector sum of $a_{i}$ and a scalar sum of $\epsilon_{i}$, respectively, over the discrete spectrum. As in Akmaev et al. (1992), in this study we employ the same isotropic wave spectrum configuration consisting of a total of 24 monochromatic waves ( 3 waves propagating in each of 8 azimuths). Unlike in that work, however, the effective vertical flux at the lower boundary, $F_{i 0}$, is allowed to vary with latitude. As mentioned, Eckermann (1995) demonstrated the importance of background temperature variations in seasonal variations of gravity-wave activity in the middle atmosphere. In this study we also fully account for the background temperature profile in integration of Eq. 2 and in calculations of the static stability parameter $N$.

\subsection{Parameterization of eddy mixing}

Due to comparatively short vertical wavelengths of the diurnal-tide modes, eddy diffusion is an important dissipation mechanism in the MLT (e.g., Forbes, 1982). However, there still exists a great deal of uncertainty as to the parameterization of eddy mixing in the middle and upper atmosphere. Akmaev et al. (1992) prescribed the eddy-diffusion coefficient $K$ approximately to match the observed temperatures in the middle atmosphere. Akmaev (1994) and Akmaev et al. (1996) used $K$ derived from the global thermal balance. In this study $K$ is determined more consistently depending on the gravity-wave energy dissipation rate and large-scale static stability.

Lilly et al. (1974) derived the following expression for the vertical eddy-diffusion coefficient in a stably stratified atmosphere

$K=c \epsilon / N^{2}$, 
where $\epsilon$ is turbulent dissipation rate. From energy balance considerations (e.g., Osborn, 1980), the maximum value of $c$ can be written in the form

$c_{\max }=\mathrm{Rf}_{\mathrm{c}} /\left(1-\mathrm{Rf}_{\mathrm{c}}\right)$,

where $\mathrm{Rf}_{\mathrm{c}}$ is the critical value for flux Richardson number. Theoretically, turbulence must collapse if $\mathrm{Rf}$ exceeds the limit of $1 / 2$ (Townsend, 1958). Atmospheric and oceanic experimental data (Lilly et al., 1974; Oakey, 1982) suggest that $\mathrm{Rf}_{\mathrm{c}} \leq 1 / 4$, and thus $c \leq 1 / 3$. It is interesting to note here how Eq. 5 compares with the Lindzen (1981) parameterization of $K$ due to wave breaking. If Eq. 4 is valid for a breaking wave, which is consistent with the approximations in the Lindzen (1981) derivation, and the complete expression for wave drag (Holton, 1982) is used, then it can be easily shown that the Lindzen formula for $K$ is equivalent to Eq. 5 with $c=1$. Assuming that the total wave energy dissipation entirely goes into turbulence, this implies $\mathrm{Rf}_{\mathrm{c}}=1 / 2$, the upper theoretical limit imposed by Townsend (1958). We conclude that the Lindzen parameterization of eddy diffusion tends to overestimate $K$, possibly due to the neglect of mechanisms other than diffusion that constrain the amplitude of breaking waves (e.g., Walterscheid and Schubert, 1990).

Obviously, Eq. 5 is not applicable for weak or unstable stratification. In the model, the unstable stratification $\left(N^{2}<0\right)$ is excluded by application of a direct convective adjustment scheme (Akmaev, 1991). Weinstock (1981) generalized Eq. 5 for the case of weak stable stratification $\left(N^{2} \rightarrow 0\right)$. The expression for $K$ derived by Weinstock essentially reduces to Eq. 5 for strong stable stratification $\left(N^{2} \gg 0\right)$, and to the famous "four-thirds law" due to Richardson (1926) (e.g., Monin and Yaglom, 1975, §24) for weak stratification. In the latter case, however, in addition to the dissipation rate $\epsilon$, specification of a characteristic length (outer scale) is required. This scale is not available from the current gravity-wave parameterization and its arbitrary specification would be equivalent to an arbitrary specification of $K$. Notably, Hines (1997) uses the Richardson law to calculate $K$ in his spectral gravity-wave parameterization wherein a characteristic vertical scale (wavenumber) is calculated as one of the pivotal parameters of the scheme.

In some middle- and upper-atmospheric models (e.g., Wu et al., 1989) weak or unstable stratification is handled using models of eddy diffusion depending on the local gradient Richardson number, Ri. These models are usually developed for the boundary layer and have the form

$K=l^{2}|\partial \mathbf{v} / \partial z| F(\mathrm{Ri})$,

where $l$ is a mixing length, typically $l=30 \mathrm{~m},|\partial \mathbf{v} / \partial z|$ is absolute value of the total vertical wind sheer. $F$ is some function of the Richardson number that is non-vanishing for $\mathrm{Ri}<\mathrm{Ri}_{\mathrm{c}}$ where $\mathrm{Ri}_{\mathrm{c}}$ is some prespecified critical Richardson number. After some experimentation, the parameterization by Levy et al. (1982) was chosen for these simulations. It has the same form as Eq. 7 and was specifically designed for middle-atmospheric tracer simulations.

Finally, turbulence must cease above the turbopause, which is of course not guaranteed by Eqs. 5 and 7. In fact, the gravity-wave energy dissipation rate, $\epsilon$, in Eq. 5 can be substantial above the turbopause due to enhanced molecular viscosity and thermal conductivity in the lower thermosphere. Hines (1997), for example, simply suggests putting $K$ to zero above the turbopause level determined from some characteristics of the gravity-wave spectrum. In this study, the following "turbopause correction" of eddy diffusion is used. Heisenberg (1948) introduced a spectral representation of turbulent diffusion due to eddies with scales smaller than $L$. For our purposes it can be written in the following form

$K(l<L)=\int_{0}^{L} f(l) d l$,

where $f(l)$ depends on the spectral distribution of turbulent energy. For the standard inertial range energy distribution, Eq. 8 reduces to the four-thirds law if $L$ is the outer scale of the inertial range. The spectral energy density, and consequently $f(l)$, sharply (exponentially) drops at scales comparable with the Kolmogorov (1941) local (inner) scale $\lambda=v^{3 / 4} \epsilon^{-1 / 4}$, where $v$ is molecular kinematic viscosity (e.g., Tatarskii, 1967, §12; Monin and Yaglom, 1975, §22). This means that eddies with scales less than $\lambda$ practically do not exist and cannot contribute to eddy diffusion. In the lower atmosphere typical scales $L \gg \lambda$ and the lower integral limit in Eq. 8 can be placed at 0 . Near the turbopause, however, $L \sim \lambda$. Formally replacing the lower integral limit in Eq. 8 by $L_{1}=c_{1} \lambda$, where $c_{1}$ is a numerical factor of the order of 1 , and assuming the standard energy density for the inertial range, we obtain the "corrected" expression

$K_{\text {corr }}=\int_{L_{1}}^{L} f(l) d l=K-c_{2} v$,

where $K$ is calculated according to Eqs. 5 or 7 and $c_{2}$ is another numerical coefficient of the order of 1 . The right-hand side in Eq. 9 is presumed positive, otherwise $K_{\text {corr }}=0$. Of course, the foregoing can only be considered a very crude approximation alternative to simply putting $K=0$ above a prespecified "turbopause level." This is not a rigorous derivation as the theory of inertial range turbulence is only applicable for very large Reynolds numbers $(L \gg \lambda)$. Otherwise the inertial range simply does not exist. A somewhat similar approach was suggested by Gavrilov and Yudin (1992).

The whole procedure of eddy diffusion calculations in the present study can now be summarized as follows. For weak stratification the parameterization of Levy et al. (1982) is used. Note that this parameterization provides different expressions for diffusivity of heat and momentum. Otherwise Eq. 5 is used with $c=1 / 3$ and $\epsilon$ assumed to equal the gravity-wave dissipation rate calculated as a sum of $\epsilon_{i}$ from Eq. 4. In this case the 
Prandtl number is simply $\operatorname{Pr}=1$, i.e., the coefficients of diffusion of heat and momentum are assumed equal. In both cases the "turbopause correction", Eq. 9, is applied with $c_{2}=1$. Since molecular viscosity $v$ is approximately inversely proportional to the atmospheric density, this correction is only important in the vicinity of turbopause at about $100 \mathrm{~km}$.

\section{Results and discussion}

Akmaev et al. (1996) presented SMLTM tidal simulations for equinox (April) conditions. A globally uniform vertical profile of the eddy-diffusion coefficient, $K(z)$, was specified from the global thermal balance (Akmaev, 1994). A uniform and isotropic gravity-wave spectrum was specified at the lower boundary. The spectrum was tuned to the CIRA-86 empirical zonal mean climatology (Fleming et al., 1990) using a procedure similar to that described by Akmaev (1997). The following gravitywave spectrum parameters were adjusted to minimize the annual global rms temperature deviation from the empirical model: phase speeds, $c_{i}$; effective vertical fluxes of horizontal momentum at the lower boundary, $F_{i 0}$; efficiency factors, $\delta_{i}$; and the horizontal wavenumber, $k$, that was assumed independent of the phase speed. In this study, for equinox conditions we use the same initial gravity-wave spectrum. At the lower boundary (100 mb), three waves are specified with phase speeds of $c_{i}=16,46$, and $47.4 \mathrm{~m} \mathrm{~s}^{-1},(i=1,2,3)$. The waves propagate in each of 8 azimuths $\beta_{j}=\pi / 8+\pi(j-1) / 4$, $(j=1, \ldots, 8)$. For each phase speed, the following effective vertical fluxes of horizontal momentum (in the direction of intrinsic phase speed) and efficiency factors are assigned: $F_{i 0}=8.4 \times 10^{-3}, 1.7 \times 10^{-4}$, and $2.6 \times 10^{-5} \mathrm{~m}^{2} \mathrm{~s}^{-2} ; \quad \delta_{i}=4 \times 10^{-3}, \quad 7.2 \times 10^{-3}, \quad$ and $3.9 \times 10^{-2},(i=1,2,3)$. The gravity-wave scheme has been modified to account for variations of the background-temperature profile in addition to variations of the background wind. The vertical eddy diffusion coefficient is now calculated as described in Sect. 3.2.
The model has been integrated for 60 days for perpetual equinox (April) and solstice (January) conditions starting from a zonally symmetric state. Wu et al. (1989) demonstrated that the diurnal migrating (propagating westward) component corresponding to the zonal wavenumber $m=1$ exceeds other diurnal components by a factor of up to $10^{3}$ in energy content at subtropical latitudes. The wind and temperature amplitudes for $m=1$ at the end of the 60-day period are presented in this study.

McLandress et al. (1996) combined wind observations at 90-114 $\mathrm{km}$ where both daytime and nighttime WINDII data are available for March-April 1992/93. The superposition of data for 4 months provided a local time coverage of at least $18 \mathrm{~h}$ between latitudes $\pm 40^{\circ}$. This facilitated reliable estimation of the diurnal wind component. Figure 1 compares diurnal amplitudes for the meridional wind with simulations for equinox conditions (April). As in the previous work (Akmaev et al., 1996), the simulations reproduce the observed features of the meridional wind-component quite well. There is general agreement in maximum amplitudes (up to about $70 \mathrm{~m} \mathrm{~s}^{-1}$ ) and in the position of the maxima both in latitude and altitude (see also, Hays et al., 1994). Akmaev et al. (1996) also noted reasonable agreement in phase for the diurnal meridional-wind component.

The observed zonal-wind amplitudes (Fig. 2) are generally smaller. The $20-\mathrm{m} \mathrm{s}^{-1}$ contour is reproduced well, at least at low latitudes. However, within this contour, the observed amplitudes are smaller by about 10-15 $\mathrm{m} \mathrm{s}^{-1}$. The difference can probably be attributed to the inadequate description of gravity-wave-induced dissipation by the simple isotropic spectrum used in the simulations. In particular, the gravity-wave spectra observed in the lower atmosphere are often anisotropic. This, in addition to the filtering by background winds in the middle atmosphere, may result in a highly anisotropic wave drag in the upper mesosphere and lower thermosphere. Also, as noted by Hays et al. (1994), estimates of tidal winds from UARS measurements are

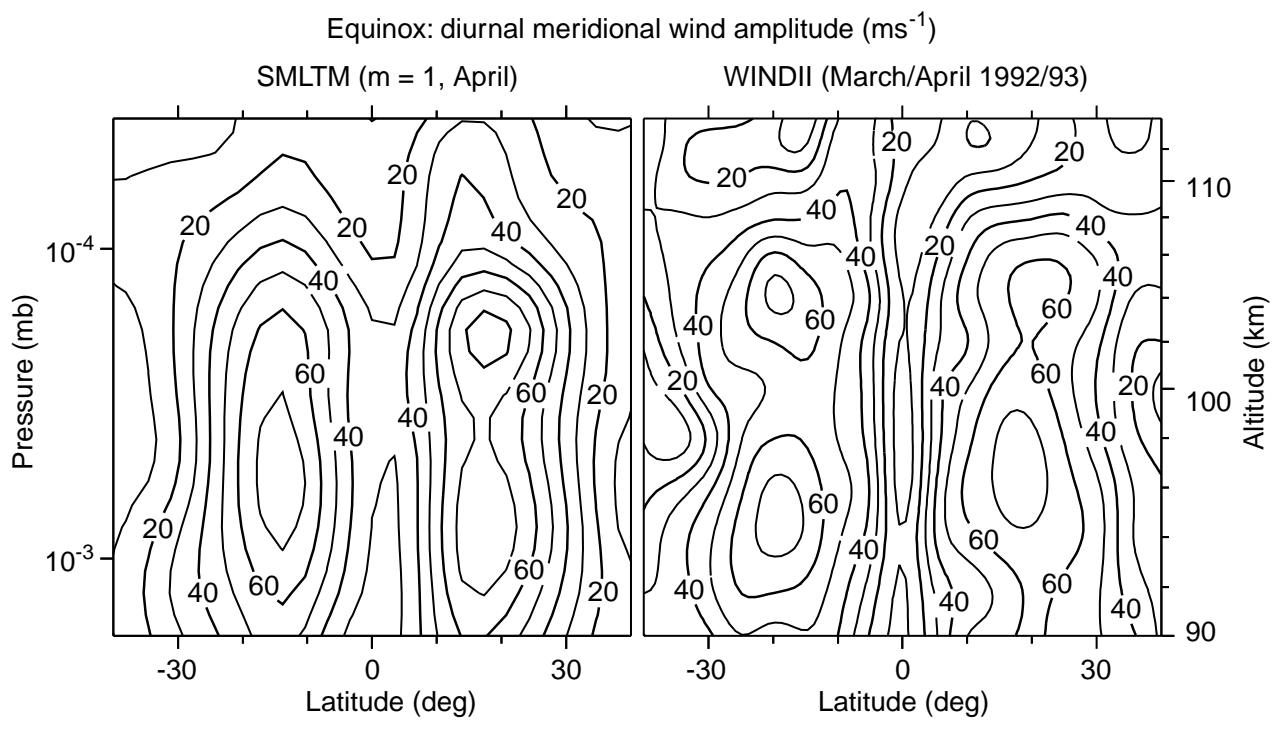

Fig. 1. Diurnal tidal amplitude for meridional wind (contour interval $\left.10 \mathrm{~m} \mathrm{~s}^{-1}\right)$ : SMLTM simulations $(m=1)$ for April (left panel); WINDII diurnal tidal amplitude (McLandress et al., 1996) for March/April 1992/93 (right panel) 


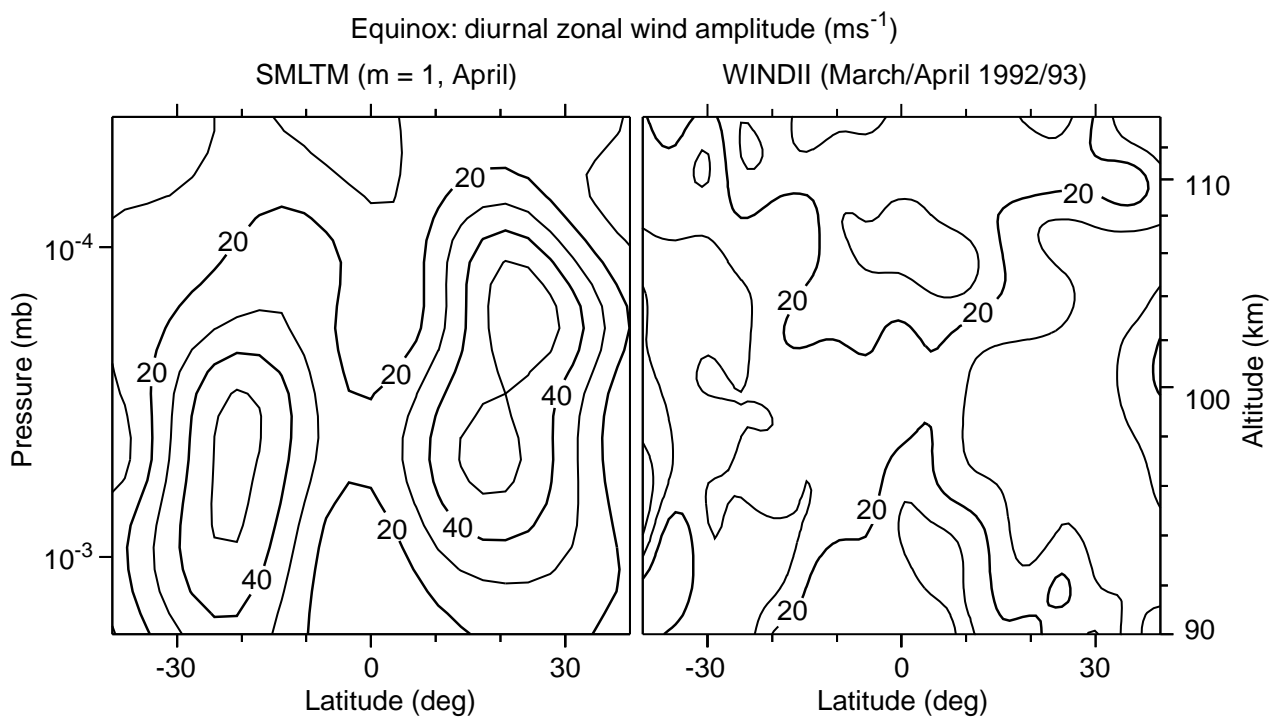

Fig. 2. Same as in Fig. 1 but for the zonal-wind component less reliable for the zonal component than for the meridional component due to comparatively strong contributions by zonal mean winds.

Akmaev et al. (1996) argued that the availability of simultaneous temperature and wind observations can be important for tidal studies in the MLT. First, independent temperature measurements can be used in validation of wind measurements. Roughly speaking, higher tidal-temperature amplitudes should correspond to higher tidal-wind amplitudes. The relation between, say, maximum temperature and wind amplitudes can be approximately estimated either from the polarization relations of the classical tidal theory (Chapman and Lindzen, 1970) or from numerical simulations. Second, temperature observations can be instrumental in clarifying the relative importance of various tidal dissipation mechanisms. For example, for the diurnal tide to become convectively unstable (cf., Wu et al., 1989; Miyahara et al., 1993), its amplitude should exceed 50$60 \mathrm{~K}$. Direct temperature observations can demonstrate whether such large amplitudes can be reached in the MLT region.

Temperatures have recently been derived from HRDI measurements in the MLT. These measurements consist of brightnesses produced by emission lines in the $\mathrm{O}_{2}\left({ }^{1} \Sigma\right)$ atmospheric A (0-0) band while viewing the limb of the earth along lines of sight with tangent heights that range from 65 to $105 \mathrm{~km}$ at $2.5-\mathrm{km}$ intervals. The measured brightnesses of these lines are related to both the band volume emission rate and the rotational temperature of the $\mathrm{O}_{2}$ molecules. Thus, in order to recover both the temperature and volume emission rate from the measurements, it is required to make observations of two different lines in the A band from the same volume of atmosphere. The lines chosen have brightnesses which change at different rates with the temperature, and the instrument is alternately tuned to each of them from one limb scan to the next. It is assumed that the volume emission rate and temperatures remain essentially constant between scans, which are separated by about

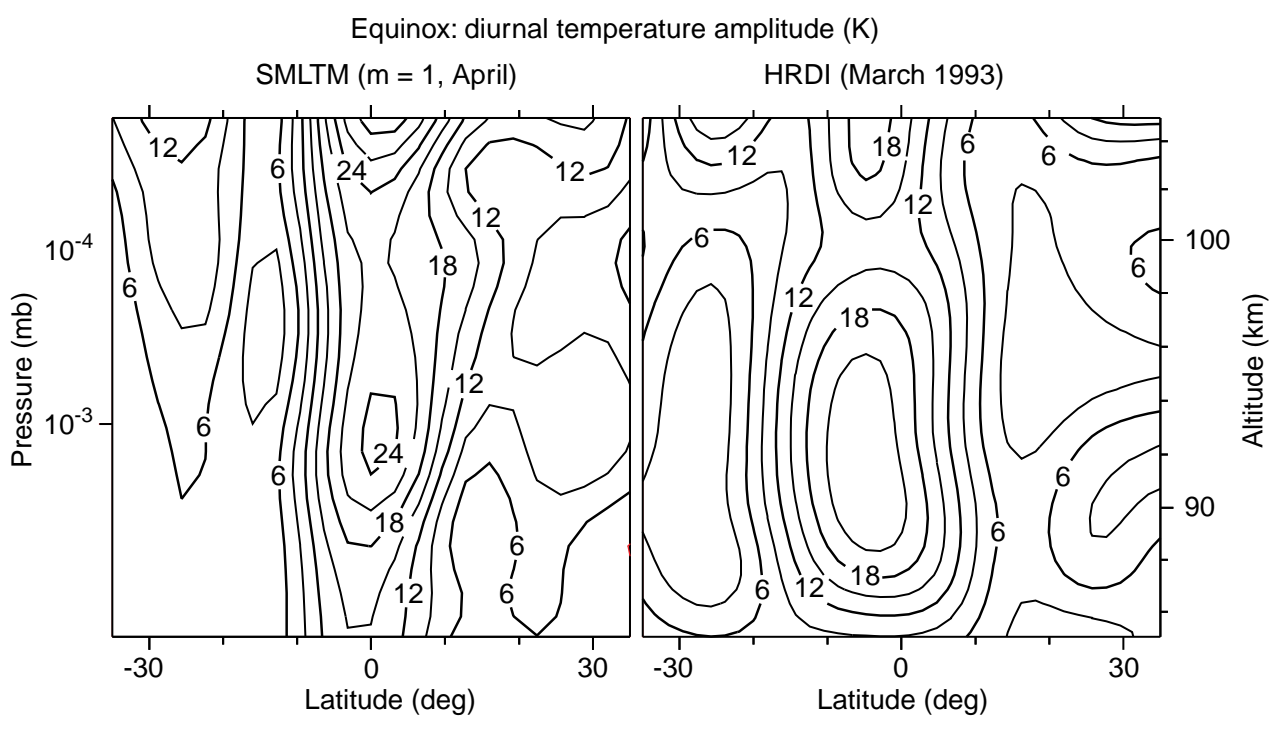

Fig. 3. Diurnal tidal amplitude for temperature (contour interval $3 \mathrm{~K})$ : SMLTM simulations $(m=1)$ for April (left panel); HRDI diurnal tidal amplitude for March 1993 (right panel) 
$200 \mathrm{~km}$ along the tangent point track. Further details of the inversion method can be found in the paper by Ortland et al. (1997).

Preliminary analysis of the temperatures measured by HRDI during 1993/94 shows a noticeable interannual variability of the monthly mean composites binned in local solar time/latitude/altitude. The temperature variations are generally consistent with weaker tidal wind amplitudes in 1994 compared to 1993 (e.g., Hays et al., 1994; Burrage et al., 1995). It is interesting to note, however, that this correspondence is not so evident for temperature oscillations in the equatorial region around equinoxes in 1994. For this reason, and since our equinox simulations compare well with the WINDII wind measurements in 1992/93 (Figs. 1 and 2), we present in this paper only the preliminary estimates of the diurnal temperature amplitudes for March and January 1993.

Figure 3 compares the SMLTM diurnal temperature amplitude for April with preliminary estimates from HRDI observations in March 1993. The experimental data have been binned in $10^{\circ}$ intervals in latitude and the diurnal amplitude has been obtained by a least-squares fit of a diurnal sinusoidal wave, implying that the diurnal signal is dominant in the MLT at low latitudes. Similar diurnal temperature amplitude distributions have been obtained for March 1994 and April 1993/94.

The temperature measurements are only available from daytime data with local time coverage of 8-12 h between latitudes $\pm 35^{\circ}$. This coverage is well known (e.g., Crary and Forbes, 1983) to be insufficient for reliable separation of the diurnal and semidiurnal tidal components and the daily mean. To attain some degree of confidence in these preliminary estimates of the diurnal temperature amplitudes, some additional independent information on the temperature distribution in the MLT has been invoked in the least-squares fits. Comparisons of globally averaged HRDI temperatures for 1993 with the CIRA-86 (Fleming et al., 1990) and MSISE-90 (Hedin, 1991) empirical models show a good agreement at $85-105 \mathrm{~km}$. Therefore, the daily mean component has been estimated using the MSISE-90 temperatures for the corresponding season. The temperature amplitudes have additionally been tested for consistency with the WINDII measurements of the meridional wind. A linear mechanistic tidal model has been used to estimate tidal dissipation from the WINDII wind measurements (Yudin et al., 1997). The diurnal tidal temperature oscillations estimated from the HRDI measurements have been found to be in reasonable agreement both in amplitude and phase with the mechanistic model simulations in the equatorial region.

Given the preliminary character of these estimates and the fact that there has been no special tuning of dissipative processes (e.g., $\mathrm{Pr}=1$ ), a generally good agreement between the SMLTM simulations and observations can be seen in Fig. 3. Both the model and observations show an equatorial maximum of tidal temperature amplitude of about 22-24 K at 90-95 km. It is clear that although the wind amplitudes are relatively large at equinox (Figs. 1 and 2), the temper- ature amplitudes are about a factor of 2 smaller than would be necessary for onset of convective instability (cf., Wu et al., 1989; Miyahara et al., 1993).

SMLTM diurnal tidal amplitudes for January obtained with the same gravity-wave forcing as for equinox (not shown) are generally lower than those presented in Figs. 1-3. This results from a combined effect of the tidal forcing at the lower boundary and of the background atmosphere on propagation of tides and gravity waves. However, the simulated peak meridional-wind and temperature amplitudes in the MLT are still higher than the amplitudes inferred from observations by up to 10-20 $\mathrm{m} \mathrm{s}^{-1}$ and 5-10 K, respectively. Numerical experiments with enhanced gravity-wave forcing at solstice have been also performed. The corresponding results are presented here.

The small-scale gravity-wave energy (e.g., Nakamura et al., 1996) and related eddy mixing (e.g., Fukao et al., 1994) in the MLT exhibit a strong seasonal cycle with solstitial maxima. Allen and Vincent (1995) also found strong seasonal variations of total gravity-wave energy density in the lower stratosphere with maxima at solstices. The maximum of total wave energy in December-January exceeds the equinoctial level by about $50-60 \%$ at low latitudes. The maximum contribution to the wave energy spectra obtained by Allen and Vincent (1995) comes from waves with comparatively short vertical wavelengths of about $2.5 \mathrm{~km}$ and intrinsic phase speeds of about 5-10 $\mathrm{m} \mathrm{s}^{-1}$. These waves are not represented in the discrete spectrum used in the present simulations. Hence, it would not be possible to specify the lower-atmospheric wave source exactly in accordance with the observations of Allen and Vincent (1995). Instead, the following simple form has been used for the effective vertical flux of horizontal momentum at the lower boundary at solstice (January)

$F_{i 0}^{s}=F_{i 0}^{e}[1+A \cos (B \phi)]$, if $\cos (B \phi)>0$,

$F_{i 0}^{s}=F_{i 0}^{e}$, otherwise.

Here $\phi$ is latitude and superscripts $s$ and $e$ refer to solstice and equinox conditions, respectively. The efficiency factors $\delta_{i}$ in Eq. 3 have been scaled the same way. Below, the results of simulations for January are presented for $A=0.5$ and $B=3$. These numerical coefficients mimic the relative $50 \%$ increase of total gravity-wave energy at low latitudes in January in general agreement with the climatology by Allen and Vincent (1995).

Figures 4 and 5 compare diurnal tidal wind amplitudes of McLandress et al. (1996) for December 1992/ 93, and January 1993/94, with SMLTM simulations for January.

Both the meridional and zonal components exhibit a fairly reasonable agreement in the magnitude and position of maximum amplitudes in the MLT region. Even with the simple representation of seasonal variations of the gravity-wave spectrum from equinox to solstice used in these simulations (Eq. 10), the model is capable of reproducing the observed decrease in maximum meridional- and zonal-wind amplitudes to about 


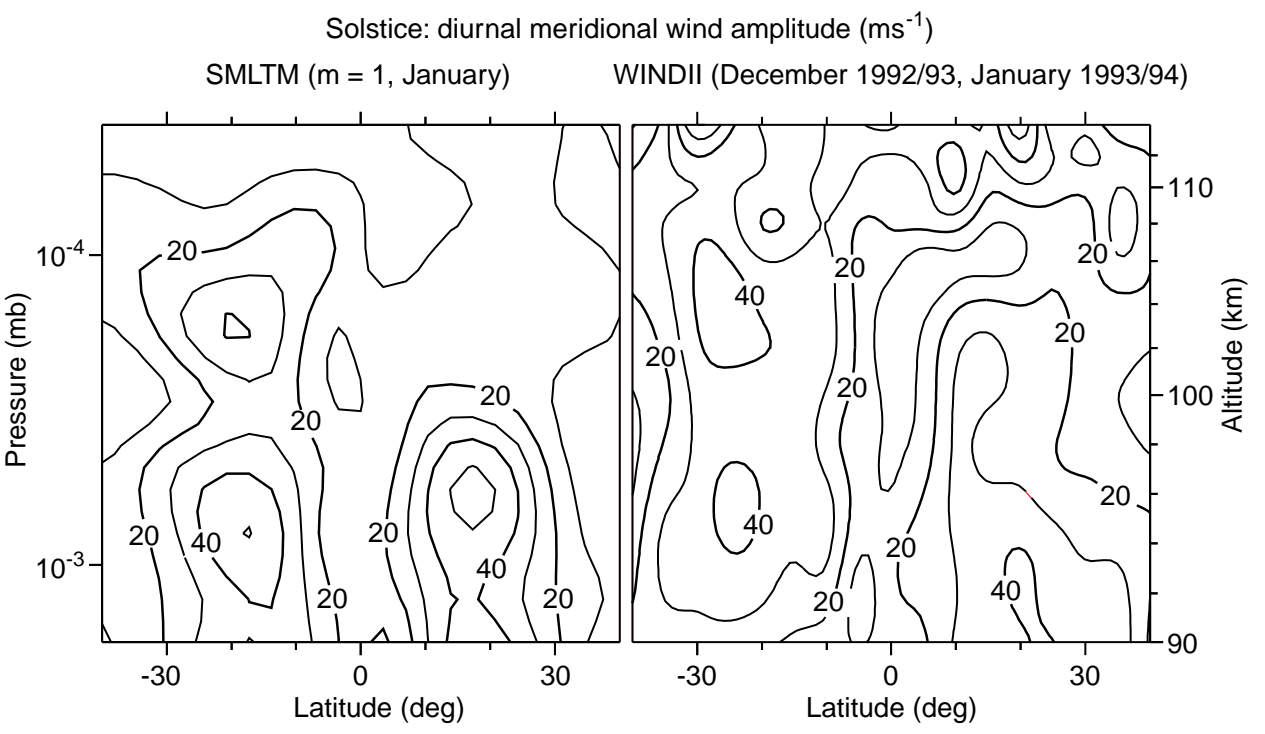

Fig. 4. Diurnal tidal amplitude for meridional wind (contour interval $\left.10 \mathrm{~m} \mathrm{~s}^{-1}\right)$ : SMLTM simulations $(m=1)$ for January (left panel); WINDII diurnal tidal amplitude (McLandress et al., 1996) for December 1992/93, and January 1993/ 94 (right panel)
40 and $30 \mathrm{~m} \mathrm{~s}^{-1}$, respectively. The simulations and observations (Hays et al., 1994; McLandress et al., 1996) also show a less symmetric character about the equator of tidal wind amplitudes at solstice as compared to equinox. This suggests enhanced excitation of asymmetric diurnal modes at solstice as well as possible filtering effects due to the background winds and dissipation.

Finally, Fig. 6 compares SMLTM diurnal temperature amplitudes for January with estimates made from HRDI temperature measurements in January 1993. A good agreement in temperature amplitudes can be seen at low latitudes. (The comparatively high temperature amplitudes along the edges of the right panel in Fig. 6 are most likely an artifact of the fitting procedure due to the poor local time coverage and contamination by the semidiurnal oscillations.) Both the model and observations predict a two-fold decrease in temperature amplitudes associated with the enhanced dissipation due to gravity-wave drag and eddy mixing.

\section{Conclusions}

Numerical simulations of the diurnal tide in the MLT region with a new Spectral mesosphere/lower thermosphere model are presented. The diurnal tide is generated in situ by the absorption of solar UV radiation. The important tidal forcing in the lower atmosphere is introduced via the lower boundary condition specified according to independent self-consistent tidal simulations with the linear Global-scale wave model. Tidal dissipation processes in the MLT are represented by a discrete spectrum gravity-wave parameterization and vertical eddy mixing. The gravity-wave scheme was originally tuned to the zonal mean climatology in the middle atmosphere. The vertical eddy-diffusion coefficient is calculated self-consistently depending on the gravity-wave dissipation rate and large-scale background stability.

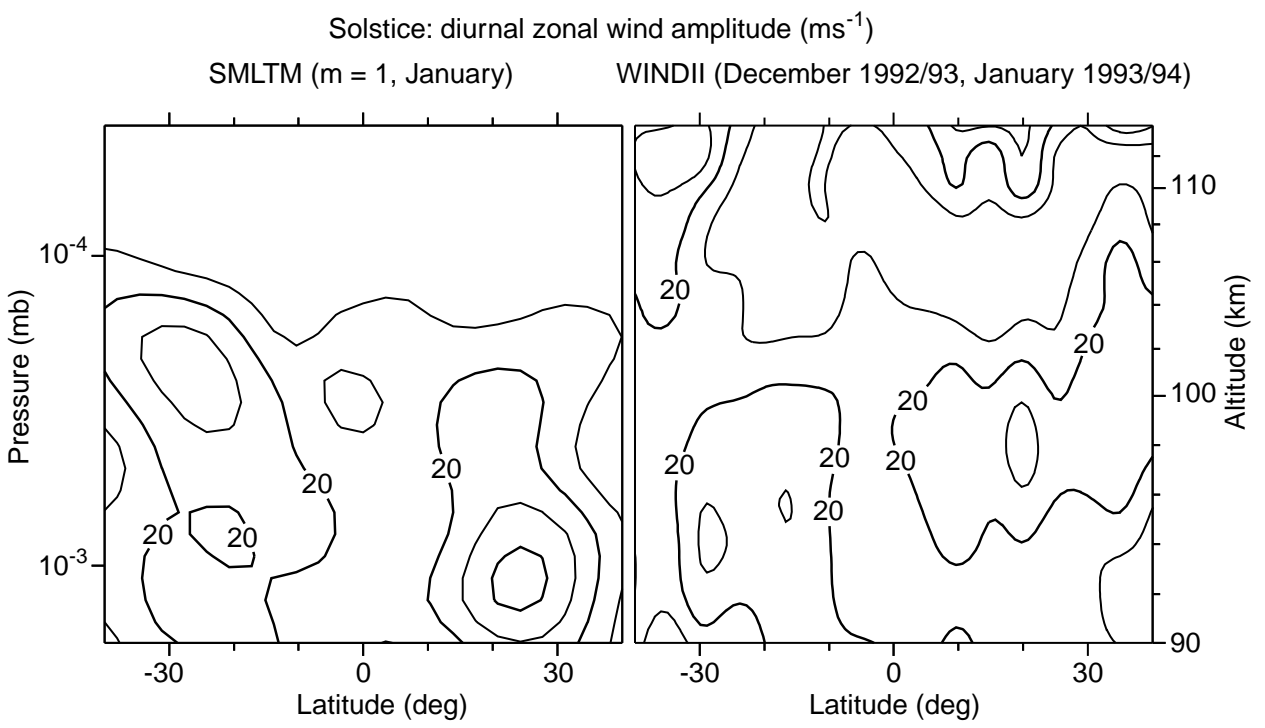

Fig. 5. Same as in Fig. 4 but for the zonal-wind component 


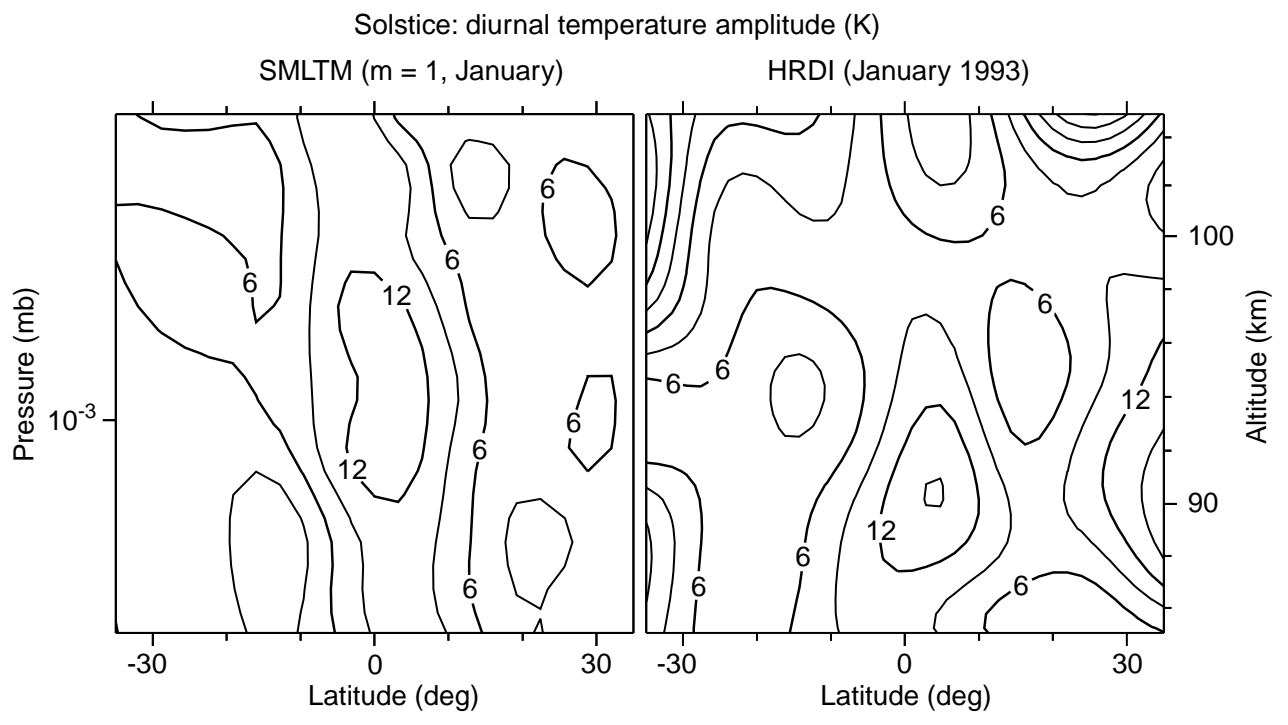

Fig. 6. Diurnal tidal amplitude for temperature (contour interval 3 $\mathrm{K})$ : SMLTM simulations $(m=1)$ for January (left panel); HRDI diurnal tidal amplitude for January 1993 (right panel)
The simulations are compared with the diurnal tidal amplitudes of winds and temperature obtained from measurements by the HRDI and WINDII instruments on board UARS. The observations of MLT winds and temperatures reveal strong seasonal variations of tidal amplitudes with maxima at equinoxes and minima at solstices. Independent observations in the lower atmosphere suggest that the total gravity-wave energy density also undergoes a strong seasonal cycle with solstitial maxima. It is demonstrated that if the gravity-wave forcing at the lower boundary is specified consistently with the gravity-wave observations, the model is capable of reproducing the gross features of seasonal variations of the diurnal tide in the MLT. The simulated amplitudes of meridional wind, both for equinox and solstice, and of zonal-wind at solstice agree well with the observations. Although the simulated maximum zonalwind amplitudes at equinox exceed observations by up to $10-15 \mathrm{~m} \mathrm{~s}^{-1}$, the availability of independent estimates of the diurnal temperature amplitudes and their close agreement with simulations provide an additional degree of confidence in the model results.

Gravity-wave drag and associated eddy mixing have long been recognized as a key factor in formation of the zonal mean climatology in the middle and upper atmosphere. The results presented in this study show that these processes also control dissipation and propagation of tidal waves into the MLT region. However, there still exists a great deal of uncertainty in the parameterization of gravity-wave spectra and eddy diffusivity in the middle and upper atmosphere. In particular, there is need for an adequate description of vertical eddy mixing applicable both to strong and weak stratification and consistent with gravity-wave energy deposition. Recent climatological studies of gravitywave spectra provide information on seasonal variations of total wave forcing in the lower atmosphere. Unfortunately, this information cannot be directly incorporated into the discrete-spectrum gravity-wave parameterization used in this study. Gravity-wave schemes based on the continuous spectrum formulation (e.g., Hines, 1997) appear to be more suitable for a universal description of eddy mixing. They can also accommodate observations of gravity-wave forcing in the lower atmosphere more consistently and thus seem to offer an attractive alternative to discrete wave spectrum parameterizations.

Acknowledgements. We thank Charles McLandress and Gordon Shepherd for providing the WINDII data and for useful feedback and suggestions. Helpful comments by Mark Burrage, Jeff Forbes, Maura Hagan, and two anonymous reviewers are also appreciated. This work has been supported by the National Science Foundation under Grants ATM-9415874 and ATM-9523951 and by the National Aeronautics and Space Administration under the UARS Program, Contract NAS5-27751, and Grant NAG5-3180.

Topical Editor F. Vial thanks U. Berger and H. G. Mayr for their help in evaluating this paper.

\section{References}

Akmaev, R. A., A direct algorithm for convective adjustment of the vertical temperature profile for an arbitrary critical lapse rate, Mon. Weather Rev., 119, 2499-2504, 1991.

Akmaev, R. A., Diagnostics and simulation of an annual cycle in the middle atmosphere, J. Geophys. Res., 99, 18933-18950, 1994.

Akmaev, R. A., Optimization of a middle atmosphere diagnostic scheme, J. Atmos. Sol.-Terr. Phys., 59, in press, 1997.

Akmaev, R. A., V. I. Fomichev, N. M. Gavrilov, and G. M. Shved, Simulation of the zonal mean climatology of the middle atmosphere with a three-dimensional spectral model for solstice and equinox conditions, J. Atmos. Terr. Phys., 54, 119-128, 1992.

Akmaev, R. A., J. M. Forbes, and M. E. Hagan, Simulation of tides with a spectral mesosphere/lower thermosphere model, Geophys. Res. Lett., 23, 2173-2176, 1996.

Allen, S. J., and R. A. Vincent, Gravity wave activity in the lower atmosphere: Seasonal and latitudinal variations, J. Geophys. Res., 100, 1327-1350, 1995.

Apruzese, J. P., M. R. Schoeberl, and D. F. Strobel, Parameterization of IR cooling in a middle atmosphere dynamics model 1. 
Effects on the zonally averaged circulation, J. Geophys. Res., 87, 8951-8966, 1982.

Banks, P. M., and G. Kockarts, Aeronomy, Part B, Academic Press, New York, 355 pp., 1973.

Bilitza, D., (Ed.), International reference ionosphere 1990, NSSDC/ WDC-A-R\&S 90-22, National Space Science Data Center World Data Center A for Rockets and Satellites, Greenbelt, Md., 1990.

Bourke, W., B. McAvaney, K. Puri, and R. Thurling, Global modeling of atmospheric flow by spectral methods, Meth. Comp. Phys., 17, 267-324, 1977.

Burrage, M. D., M. E. Hagan, W. R. Skinner, D. L. Wu, and P. B. Hays, Long-term variability in the solar diurnal tide observed by HRDI and simulated by GSWM, Geophys. Res. Lett., 22, 2641-2644, 1995.

Chan, K. L., H. G. Mayr, J. G. Mengel, and I. Harris, A spectral approach for studying middle and upper atmospheric phenomena, J. Atmos. Terr. Phys., 56, 1399-1419, 1994.

Chapman, S., and R. S. Lindzen, Atmospheric tides, thermal and gravitational, Gordon and Breach, New York, 200 pp., 1970.

Crary, D. J., and J. M. Forbes, On the extraction of tidal information from measurements covering a fraction of a day, Geophys. Res. Lett., 10, 580-582, 1983.

Eckermann, S. D., On the observed morphology of gravity-wave and equatorial-wave variance in the stratosphere, J. Atmos. Terr. Phys., 57, 105-134, 1995.

Fleming, E. L., S. Chandra, J. J. Barnett, and M. Corney, Zonal mean temperature, pressure, zonal wind and geopotential height as functions of latitude, Adv. Space Res., 10, (12)11-(12)59, 1990.

Fomichev, V. I., and G. M. Shved, Parameterization of the radiative flux divergence in the $9.6 \mu \mathrm{m} \mathrm{O}_{3}$ band, J. Atmos. Terr. Phys., 47, 1037-1049, 1985

Fomichev, V. I., A. A. Kutepov, R. A. Akmaev, and G. M. Shved, Parameterization of the $15 \mu \mathrm{m} \mathrm{CO} 2$ band cooling in the middle atmosphere (15-115 km), J. Atmos. Terr. Phys., 55, 7-18, 1993.

Forbes, J. M., Atmospheric tides 1. Model description and results for the solar diurnal component, J. Geophys. Res., 87, 5222$5240,1982$.

Forbes, J. M., Tidal and planetary waves, in The upper mesosphere and lower thermosphere: A review of experiment and theory, Eds. R. M. Johnson and T. L. Killeen, AGU, 67-87, 1995.

Fukao, S., M. D. Yamanaka, N. Ao, W. K. Hocking, T. Sato, M. Yamamoto, T. Nakamura, T. Tsuda, and S. Kato, Seasonal variability of vertical eddy diffusivity in the middle atmosphere: 1. Three-year observations by the middle and upper atmosphere radar, J. Geophys. Res., 99, 18973-18987, 1994.

Gavrilov, N. M., Parameterization of accelerations and heat flux divergences produced by internal gravity waves in the middle atmosphere, J. Atmos. Terr. Res., 52, 707-713, 1990.

Gavrilov, N. M., and V. A. Yudin, Model for coefficients of turbulence and effective Prandtl number produced by breaking gravity waves in the upper atmosphere, J. Geophys. Res., 97, 7619-7624, 1992

Groves, G. V., Hough components of ozone heating, J. Atmos. Terr. Phys., 44, 111-121, 1982a.

Groves, G. V., Hough components of water vapor heating, J. Atmos. Terr. Phys., 44, 281-290, 1982 b.

Hagan, M. E., Comparative effects of migrating solar sources on tidal signatures in the middle and upper atmosphere, J. Geophys. Res., 101, 21213-21222, 1996.

Hagan, M. E., J. M. Forbes, and F. Vial, On modeling migrating solar tides, Geophys. Res. Lett., 22, 893-896, 1995.

Hays, P. B., D. L. Wu, and the HRDI science team, Observations of the diurnal tide from space, J. Atmos. Sci., 51, 3077-3093, 1994.

Hedin, A. E., Extension of the MSIS thermosphere model into the middle and lower atmosphere, J. Geophys. Res., 96, 1159-1172, 1991.

Heisenberg, W., Zur statistischen Theorie der Turbulenz, Z. Phys., 124, 628-657, 1948.
Hines, C. O., Doppler-spread parameterization of gravity-wave momentum deposition in the middle atmosphere. Part 1: Basic formulation, J. Atmos. Sol.-Terr. Phys., 59, 371-386, 1997.

Holton, J. R., The role of gravity wave induced drag and diffusion in the momentum budget of the mesosphere, J. Atmos. Sci., 39, 791-799, 1982.

Holton, J. R., The influence of gravity wave breaking on the general circulation of the middle atmosphere, J. Atmos. Sci., 40, 2497-2507, 1983.

Khattatov, B. V., M. A. Geller, V. A. Yudin, and P. B. Hays, Diurnal migrating tide as seen by the high-resolution Doppler imager/UARS. 2. Monthly mean global zonal and vertical velocities, pressure, temperature and inferred dissipation $J$. Geophys. Res., 102, 4423-4435, 1997.

Kolmogorov, A. N., Local structure of turbulence in an incompressible viscous fluid at very large Reynolds numbers, Doklady Acad. Sci. USSR, 30 299-301, 1941.

Levy, H., II, J. D. Mahlman, and W. J. Moxim, Tropospheric $\mathrm{N}_{2} \mathrm{O}$ variability, J. Geophys. Res., 87, 3061-3080, 1982.

Lilly, D. K., D. E. Waco, and S. I. Adelfang, Stratospheric mixing estimated from high-altitude turbulence measurements, J. Appl. Meteorol, 13, 488-493, 1974.

Lindzen, R. S., Turbulence and stress owing to gravity wave and tidal breakdown, J. Geophys. Res., 86, 9707-9714, 1981.

Matsuno, T., A quasi one-dimensional model of the middle atmosphere circulation interacting with internal gravity waves, J. Meteorol. Soc. Japan, 60, 215-226, 1982.

McLandress, C., Y. Rochon, G. G. Shepherd, B. H. Solheim, G. Thuillier, and F. Vial, The meridional wind component of the thermospheric tide observed by WINDII on UARS, Geophys. Res. Lett., 21, 2417-2420, 1994.

McLandress, C., G. G. Shepherd, and B. H. Solheim, Satellite observations of thermospheric tides: Results from the Wind Imaging Interferometer on UARS, J. Geophys. Res., 101, 40934114, 1996.

Mengel, J. G., H. G. Mayr, K. L. Chan, C. O. Hines, C. A. Reddy, N. F. Arnold, and H. S. Porter, Equatorial oscillations in the middle atmosphere generated by small-scale gravity waves, Geophys. Res. Lett., 22, 3027-3030, 1995.

Miyahara, S., Y. Yoshida, and Y. Miyoshi, Dynamic coupling between the lower and upper atmosphere by tides and gravity waves, J. Atmos. Terr. Phys., 55, 1039-1053, 1993.

Monin, A. S., and A. M. Yaglom, Statistical fluid mechanics: Mechanics of turbulence, v. 2, MIT Press, Cambridge, Ma., 874 pp., 1975.

Nakamura, T., T. Tsuda, S. Fukao, A. H. Manson, C. E. Meek, R. A. Vincent, and I. M. Reid, Mesospheric gravity waves at Saskatoon $\left(52^{\circ} \mathrm{N}\right)$, Kyoto $\left(35^{\circ} \mathrm{N}\right)$, and Adelaide $\left(35^{\circ} \mathrm{S}\right), J$. Geophys. Res., 101, 7005-7012, 1996.

Oakey, N. S., Determination of the rate of dissipation of turbulent energy from simultaneous temperature and velocity shear microstructure measurements, J. Phys. Oceanogr., 12, 256-271, 1982.

Ortland, D. A., P. B. Hays, W. R. Skinner, and J.-H. Yee, Remote sensing of mesospheric temperature and $\mathrm{O}_{2}\left({ }^{1} \Sigma\right)$ band volume emission rates with the High-Resolution Doppler Imager, $J$. Geophys. Res., 102, submitted, 1997.

Osborn, T. R., Estimates of the local rate of vertical diffusion from dissipation measurements, J. Phys. Oceanogr., 10, 83-89, 1980.

Plumb, R. A., and A. D. McEwan, The instability of a forced standing wave in a viscous stratified fluid: A laboratory analogue of the quasi-biennial oscillation, J. Atmos. Sci., 35, 1827-1839, 1978.

Prusa, J. M., P. K. Smolarkiewicz, and R. R. Garcia, Propagation and breaking at high altitudes of gravity waves excited by tropospheric forcing, J. Atmos. Sci., 53, 2186-2216, 1996.

Richardson, L. F., Atmospheric diffusion shown on a distanceneighbour graph, Proc. R. Soc. London, 110, 709-737, 1926.

Shine, K. P., and J. A. Rickaby, Solar radiative heating due to absorption by ozone, in Ozone in the atmosphere, Eds. R. D. Bojkov and P. Fabian, Deepak, Hampton, Va., pp. 597-600, 1989. 
Strobel, D. F., Parameterization of the atmospheric heating rate from 15 to $120 \mathrm{~km}$ due to $\mathrm{O}_{2}$ and $\mathrm{O}_{3}$ absorption of solar radiation, J. Geophys. Res., 83, 6225-6230, 1978.

Tatarskii, V. I., Propagation of waves in a turbulent atmosphere, Nauka, Moscow, 548 pp., 1967.

Tobiska, W. K., Revised solar extreme ultraviolet flux model, $J$. Atmos. Terr. Phys., 53, 1005-1018, 1991.

Torr, M. R., D. G. Torr, R. A. Ong, and H. E. Hinteregger, Ionization frequencies for major thermospheric constituents as a function of solar cycle 21, Geophys. Res. Lett., 6, 771-774, 1979.

Townsend, A. A., Turbulent flow in a stably stratified atmosphere, J. Fluid Mech., 3, 361-372, 1958.
Walterscheid, R. L., and G. Schubert, Nonlinear evolution of an upward propagating gravity wave: overturning, convection, transience, and turbulence, J. Atmos. Sci., 47, 101-125, 1990.

Weinstock, J., Vertical turbulence diffusivity for weak or strong stable stratification, J. Geophys. Res., 86, 9925-9928, 1981.

Wu, D.-H., S. Miyahara, and Y. Miyoshi, A nonlinear simulation of the thermal diurnal tide, J. Atmos. Terr. Phys., 51, 10171030, 1989.

Yudin, V. A., B. V. Khattatov, M. A. Geller, D. A. Ortland, C. McLandress, and G. G. Shepherd, Thermal tides and studies to tune the mechanistic tidal model using UARS observations, Ann. Geophysicae, 15, this issue, 1997. 